\title{
Seipin deficiency in mice causes loss of dopaminergic neurons via aggregation and phosphorylation of a-synuclein and neuroinflammation
}

Ling Wang ${ }^{1,2}$, Juan Hong ${ }^{1,2}$, Yajuan $W_{u^{2}}$, George Liư ${ }^{3}$, Wenfeng $Y u^{4}$ and Ling Chen ${ }^{1,2}$

\begin{abstract}
Seipin gene is originally found in type 2 congenital generalized lipodystrophy (CGL2) to involve lipid droplet formation. Recently, decrease of seipin expression is reported in substantia nigra of Parkinson's disease patients. Dopaminergic neurons in substantia nigra pars compacta expressed the seipin protein. The objective of this study is to investigate influence of the seipin deficiency on dopaminergic neurons and motor behaviors. Neuronal seipin knockout (seipin-nKO) mice (3-12 months of age) displayed an age-related deficit in motor coordination. The number of dopaminergic neurons in seipin-nKO mice was age dependently reduced with increase in cleaved caspase-3. The levels of aSyn oligomers and oligomer phosphorylation (S129), but not aSyn monomers, were elevated in dopaminergic neurons and substantia nigra of seipin-nKO mice. The PPARY expression in seipin-nKO mice was reduced. In seipin-nKO mice, the phosphorylation of GSK3 $\beta$ was increased at Tyr216 and was reduced at Ser9, which was corrected by the PPARY agonist rosiglitazone. The increased IL-6 level in seipin-nKO mice was sensitive to rosiglitazone and GSK3B inhibitor AR-A014418. The enhanced phosphorylation of aSyn was prevented by rosiglitazone and ARA014418, while the increase in aSyn oligomers was corrected only by rosiglitazone. The treatment of seipin-nKO mice with rosiglitazone and AR-A014418 rescued the death of dopaminergic neurons, which was accompanied by the improvement of motor coordination. Therefore, the results indicate that seipin deficiency causes an age-related loss of dopaminergic neurons and impairment of motor coordination through reducing PPARY to enhance aggregation and phosphorylation of aSyn and neuroinflammation.
\end{abstract}

\section{Introduction}

Congenital generalized lipodystrophy (CGL) is an autosomal recessive disorder characterized by a neartotal loss of adipose tissue, severe insulin resistance, hypertriglyceridemia, and fatty liver ${ }^{1,2}$. Patients with CGL2 have sensorineural deafness and cognitive

Correspondence: Ling Chen (lingchen@njmu.edu.cn) or Wenfeng Yu (wenfengyu@hotmail.com)

${ }^{1}$ State Key Lab of Reproductive Medicine, Nanjing Medical University, 210029 Nanjing, China

${ }^{2}$ Department of Physiology, Nanjing Medical University, 210029 Nanjing, China

Full list of author information is available at the end of the article

These authors contributed equally: Ling Wang, Juan Hong.

Edited by A Yaron disorder ${ }^{3}$ by mutation in the Berardinelli-Seip congenital lipodystrophy 2 gene that encodes $\operatorname{seipin}^{4,5}$. Seipin has been reported in various neurological syndromes $^{6}$. Heterozygosity for missense mutations (N88S/S90L) in seipin is associated with a broad spectrum of motoneuron diseases, such as seipinopathy ${ }^{7}$. The motor neurons and peripheral motor axons are differentially affected in patients with seipin mutations $^{8,9}$. The overexpression of human seipin mutants in mice caused the death of alpha motor neurons in the spinal cord which was not associated with total loss of adipose tissue ${ }^{10}$. In contrast, the loss-

\section{(c) The Author(s) 2018}

(c) (i) Open Access This article is licensed under a Creative Commons Attribution 4.0 International License, which permits use, sharing, adaptation, distribution and reproduction c. in any medium or format, as long as you give appropriate credit to the original author(s) and the source, provide a link to the Creative Commons license, and indicate if changes were made. The images or other third party material in this article are included in the article's Creative Commons license, unless indicated otherwise in a credit line to the material. If material is not included in the article's Creative Commons license and your intended use is not permitted by statutory regulation or exceeds the permitted use, you will need to obtain permission directly from the copyright holder. To view a copy of this license, visit http://creativecommons.org/licenses/by/4.0/. 


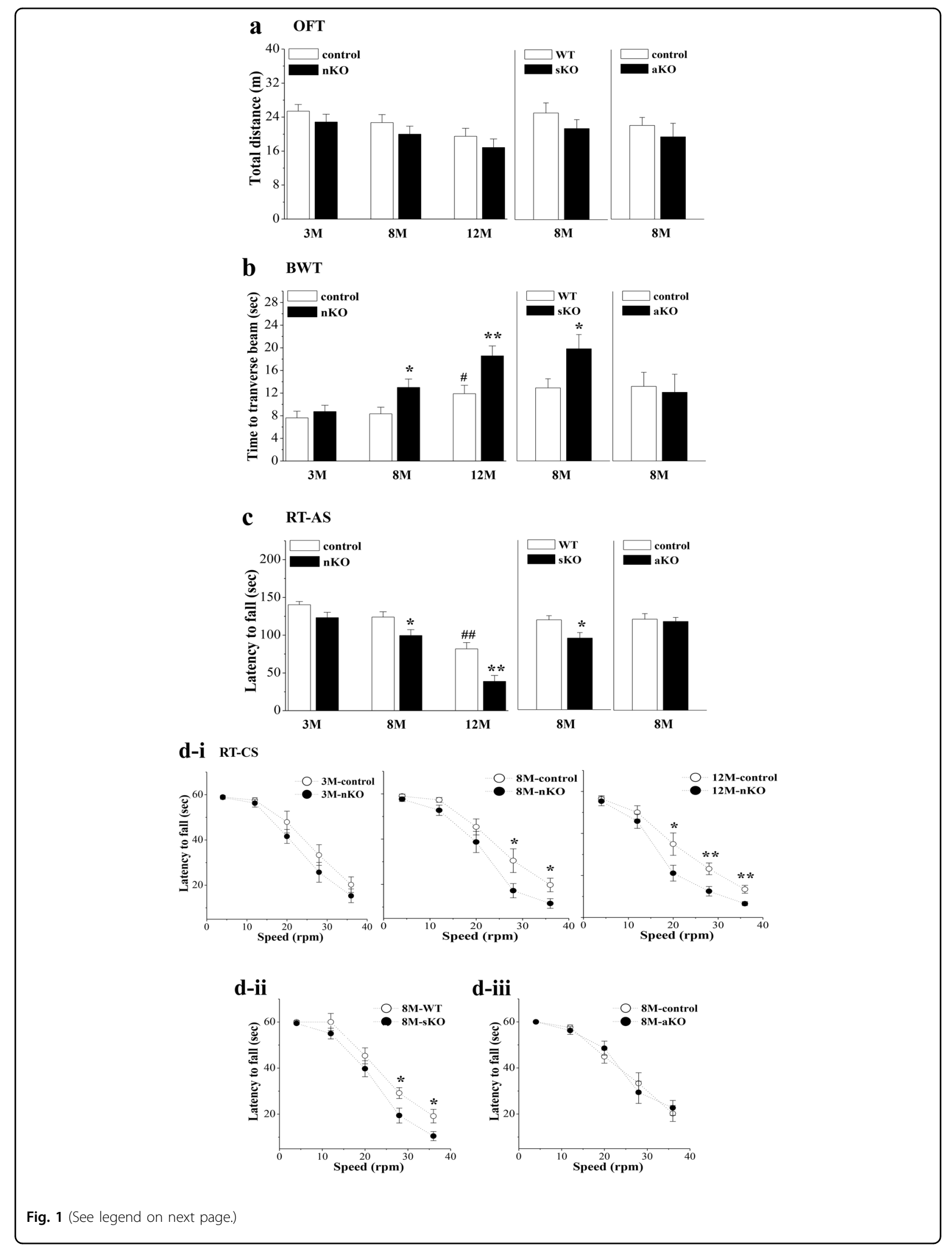


(see figure on previous page)

Fig. 1 Neuronal seipin deficiency impairs motor coordination. a Influence of neuronal seipin deficiency on spontaneous locomotion as assessed by OFT. Bar graph shows traveled distance in 3-M-old (3 M), 8-M-old (8 M), and 12-M-old (12 M) control and seipin-nKO mice; 8-M-old WT and seipinsKO mice; and 8-M-old control and seipin-aKO mice. $\mathbf{b}$ Bar graph shows time (s) to traverse the beam in BWT. ${ }^{*} P<0.05$ and ${ }^{* *} P<0.01$ vs. age-matched control mice (two-way ANOVA) or WT mice (t-test). \#P<0.05 vs. 3-M-old control mice (two-way ANOVA). c Each point represents the latency (s) on rotated rod with accelerating speed (RT-AS). ${ }^{*} P<0.05$ and ${ }^{* *} P<0.01$ vs. age-matched control mice (two-way ANOVA) or WT mice ( $t$-test). ${ }^{\# \#} P<0.01$ vs. 3-M-old control mice (two-way ANOVA). $\mathbf{d}$ Bars indicate the latency (s) on rotated rod with constant speeds (RT-CS) in control mice and seipin-nKO mice (d-i), 8-M-old WT mice and seipin-sKO mice (d-ii), and 8-M-old control mice and seipin-aKO mice (d-iii). ${ }^{*} P<0.05$ and ${ }^{* *} P<0.01$ vs. age-matched control mice or WT mice (repeated measures ANOVA)

of-function mutations in seipin gene did not cause the death of alpha motor neurons in mice; however, neuronal seipin knockout (seipin-nKO) mice at 2 months of age have a tendency to decrease the latency of falling off during the rotarod test $(\mathrm{RT})^{11}$.

The proteomic or Western blotting analysis of human demonstrated a 2.5-fold decrease of seipin expression levels in the substantia nigra (SN) of Parkinson's disease patients ${ }^{12}$. The protein was observed similarly diminished in the locus coeruleus of Parkinson's disease patients, another aminergic nucleus affected earlier than $\mathrm{SN}$ in the disease process ${ }^{13}$. Parkinson's disease is a common neurodegenerative disorder characterized by the gradual dysfunction of the extrapyramidal motor system and progressive loss of dopaminergic neurons in substantia nigra pars compacta (SNpc). Susceptible nerve cells exhibit intracellular inclusions termed Lewy bodies formed by the abnormal accumulation and aggregation of $\alpha$-synuclein $(\alpha \operatorname{Syn})^{14}$. The accumulation of oligomeric $\alpha$ Syn may cause the degeneration of dopaminergic neurons. Seipin, an endoplasmic reticulum (ER)resident membrane protein, plays a role in the generation of peroxisome proliferator-activated receptor gamma (PPAR $\gamma$ ). Seipin deficiency via reducing PPAR $\gamma$ increases glycogen synthase kinase-3 $\beta$ (GSK-3 $\beta$ ) activity, which increases in the levels of interleukin-6 (IL-6) and tumor necrosis factor- $\alpha(\mathrm{TNF}-\alpha)^{15}$. The activation of GSK-3 $\beta$ promotes inflammatory responses in the brain ${ }^{16}$. In brain of Parkinson's disease, a glial reaction increases the release of various inflammatory factors. The activation of PPAR $\gamma$ or the inhibition of GSK-3 $\beta$ has been reported to decrease $\alpha$ Syn protein expression and oligomerization $^{17,18}$. In addition, the seipin knockdown is known to cause the accumulation of phosphatidic acids in yeast ${ }^{2}$, adipose cells ${ }^{19}$, and neuronal cells ${ }^{20}$. The exposure of cultured dopaminergic neurons to physiological polyunsaturated fatty acid concentrations increases the levels of soluble $\alpha$ Syn oligomers and insoluble $\alpha$ Syn aggregates and enhances its deposition into cytoplasmic intraneuronal Lewy-like inclusions ${ }^{21}$. Therefore, it is of great interest to investigate whether the seipin deficiency causes Parkinson's disease pathogenesis.

\section{Results}

\section{Influence of seipin deficiency on motor coordination}

Spontaneous activity was initially examined using an open-field test (OFT). The group means for the total distance traveled in 3-month-old (M-old), 8-M-old, and 12-M-old seipin-nKO mice, 8-M-old seipin-sKO mice or 8-M-old seipin-aKO mice did not significantly differ from those of age-matched control mice (nestin-Cre mice) or wild-type (WT) mice $(P>0.05, n=12$; Fig. 1a). The beam walking test (BWT) (Fig. 1b) and RTs with accelerating speed (RT-AS; Fig. 1c) or constant speed (RT-CS; Fig. 1d) were used to estimate the ability of motor coordination. In seipin-nKO mice, the time that mice traversed the beam in BWT or the latency that mice remained on the rotarod before falling off were affected by genotype (BWT: $F_{(1,66)}$ $=13.472, P<0.001$; RT-AS: $F_{(1,66)}=23.029, P<0.001$; RT-CS: $\left.F_{(1,66)}=20.707, P<0.001\right)$. Compared to control mice, 8 -M-old and 12-M-old seipin-nKO mice spent a longer time traversing the beam in the BWT $(8 \mathrm{M}: P<$ 0.05; 12M: $P<0.01)$ and remained on the rotarod for less time in the RT-CS (8M: $28 \mathrm{rpm}: P<0.05,36 \mathrm{rpm}: P<0.05$; 12M: $20 \mathrm{rpm}: P<0.05,28$ or $36 \mathrm{rpm}: P<0.01)$ or RT-AS (8M: $P<0.05$; 12M: $P<0.01$ ). Although 3 -M-old seipinnKO mice had a tendency to have an increased walking time in the BWT and a decreased latency in the RT-CS or RT-AS, the group comparison with control mice failed to reach significance $(P>0.05)$. Consistently, 8-M-old seipinsKO mice required more time to traverse the beam in the BWT $(P<0.05, n=12)$ and had a decreased latency on the rotarod of RT-CS $(P<0.05, n=12)$ or RT-AS $(P<$ $0.05, n=12$ ) than WT mice. By contrast, the time spent to traverse the beam in the BWT or remain on the rotarod in the RT-CS and RT-AS was not significantly different between 8-M-old control mice (nestin-Cre mice) and seipin-aKO mice $(P>0.05, n=12)$. These results indicate that neuronal seipin deficiency causes an age-related progressive decline in motor coordination.

\section{Influence of seipin deficiency on dopaminergic neurons in the SNpc}

The western blotting analysis showed the expression of seipin protein at approximately $44 \mathrm{kD}$ in the $\mathrm{SN}$ of 3-Mold and 8-M-old control mice (Fig. 2a) and a lack of seipin 

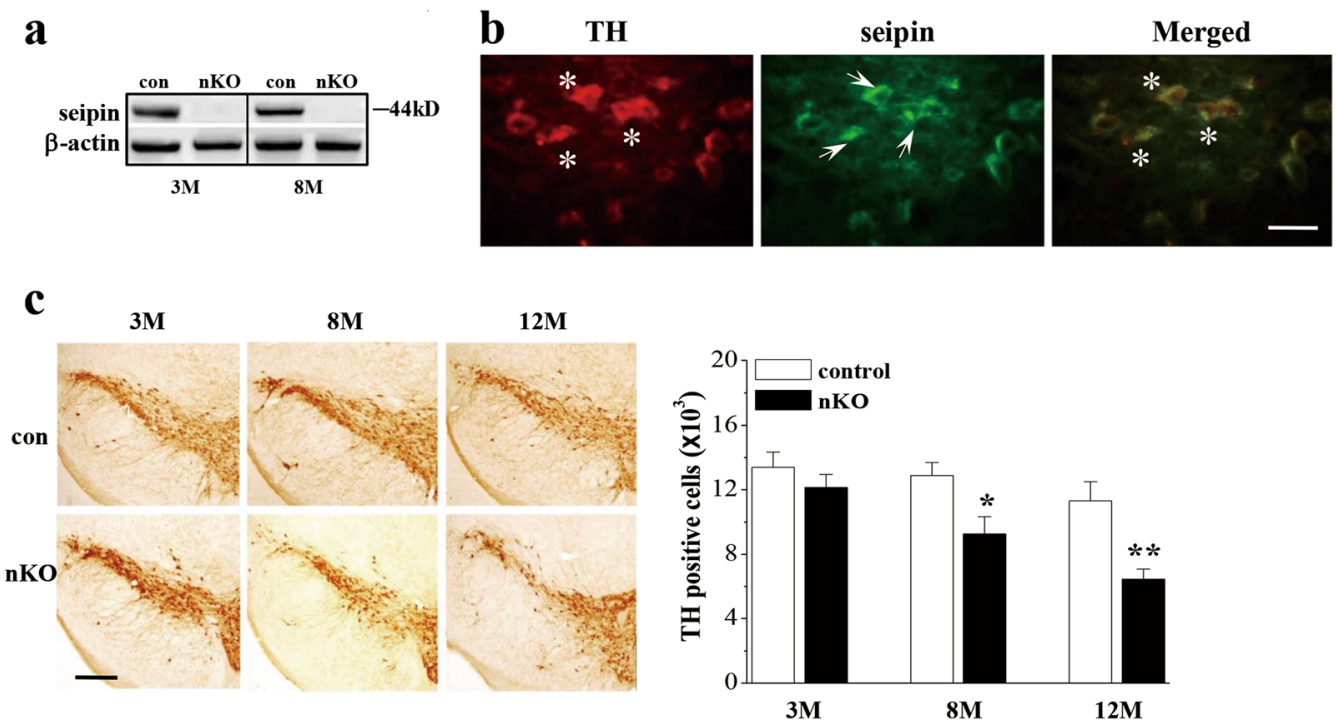

Fig. 2 Neuronal seipin deficiency causes a loss of dopaminergic neurons in the SNpc. a Levels of seipin protein in SN of 3-M-old (3 M) and 8-Mold $(8 \mathrm{M})$ control mice (con) and seipin-nKO mice (nKO). b Representative fluorescence images of seipin (green) and tyrosine hydroxylase (TH, red) double immuno-staining in SNpc of 8-M-old control mice (scale bar $=50 \mu \mathrm{m}$ ). The arrows indicate seipin-positive cells; the asterisks represent THpositive cells. c Stereological counts of TH-positive cells throughout SNpc of 3-M-old (3 M), 8-M-old (8 M), and 12-M-old (12 M) control mice and seipin-nKO mice. ${ }^{*} P<0.05$ and ${ }^{* *} P<0.01$ vs. age-matched control mice (two-way ANOVA). Representative photographs of TH staining. Scale bar $=$ $200 \mu \mathrm{m}$

protein in seipin-nKO mice. Using seipin and tyrosine hydroxylase $(\mathrm{TH})$ double immunohistochemical staining, we observed the seipin-positive dopaminergic neurons in SNpc of 8-M-old control mice (Fig. 2b).

To explore the cellular mechanisms underlying impaired motor coordination caused by seipin deficiency, we performed a stereological count of $\mathrm{TH}$ positive cells. The number of $\mathrm{TH}$-positive cells was affected by genotype $\left(F_{(1,30)}=18.480, \quad P<0.001\right.$; Fig. 2c). In comparison with control mice, the number of TH-positive cells was reduced by approximately $9 \%$ in 3-M-old seipin-nKO mice $(P>0.05), 28 \%$ in 8 -M-old seipin-nKO mice $(P<0.05)$, and $43 \%$ in $12-\mathrm{M}$-old seipin-nKO mice $(P<0.01)$. These results indicate that seipin deficiency causes an age-related progressive loss of dopaminergic neurons.

\section{Influence of seipin deficiency on oligomerization and phosphorylation of aSyn in SN}

To investigate the molecular mechanisms underlying the loss of dopaminergic neurons by seipin deficiency, the oligomerization and phosphorylation (S129) of $\alpha$ Syn were examined using non-reducing denaturing PAGE in the $\mathrm{SN}$ of 3-M-old and 8-M-old control mice and seipin-nKO mice (Fig. 3a). The levels of $\alpha$ Syn oligomers were affected by genotype $\left(F_{(1,20)}=101.019, P<0.001\right.$; Fig. 3c $)$ and genotype $\times$ age $\left(F_{(1,20)}=57.588, P<0.001\right)$. The level of $\alpha$ Syn oligomers $(70-100 \mathrm{kD})$ was higher in 8 -M-old
seipin-nKO mice than that in age-matched control mice $(P<0.01)$. Although 3 -M-old seipin-nKO mice had a tendency to increase the oligomerization of $\alpha$ Syn, the group comparison with control mice failed to reach significance $(P>0.05)$. Consistently, the increase of $\alpha$ Syn oligomers in 8-M-old seipin-nKO mice was determined by the examination of native PAGE (Fig. 3f). The analysis of reducing denaturing PAGE in seipin-nKO mice (Fig. 3g) showed a similar increase in the level of $\alpha$ Syn oligomers $(P<0.01, n=6$; Fig. $3 \mathrm{i})$. In addition, the phosphorylation of $\alpha$ Syn oligomers (p- $\alpha$ Syn) was affected by genotype $\left(F_{(1,20)}=65.58, P<0.001\right.$; Fig. $\left.3 e\right)$ and genotype $\times$ age $\left(F_{(1,20)}=23.769, P<0.001\right)$. The level of $\alpha$ Syn phosphorylation (ratio of $\mathrm{p}-\alpha$ Syn/total $\alpha$ Syn oligomers) in 3-M-old $(P<0.05)$ or 8-M-old seipin-nKO mice $(P<0.01)$ was elevated compared to that in control mice. In contrast, neither the levels of the $\alpha$ Syn monomer $(19 \mathrm{kD})(P>$ $0.05, n=6$; Fig. 3b) nor the levels of phosphorylated $\alpha$ Syn monomer $(P>0.05, n=6$; Fig. $3 \mathrm{~d})$ in seipin-nKO mice were different from those of control mice. The $\alpha$ Syn monomer by reducing denaturing PAGE showed a slight increase in seipin-nKO mice, but the group when compared with control mice failed to reach the significance $(P$ $>0.05, n=6$; Fig. 3h).

The double-immunofluorescence staining of $\alpha$ Syn phosphorylation (p- $\alpha$ Syn) and TH showed the elevation of the phosphorylated $\alpha$ Syn in TH-positive cells of 8-M-old seipin-nKO mice (Fig. 3j). By double-immunofluorescence 

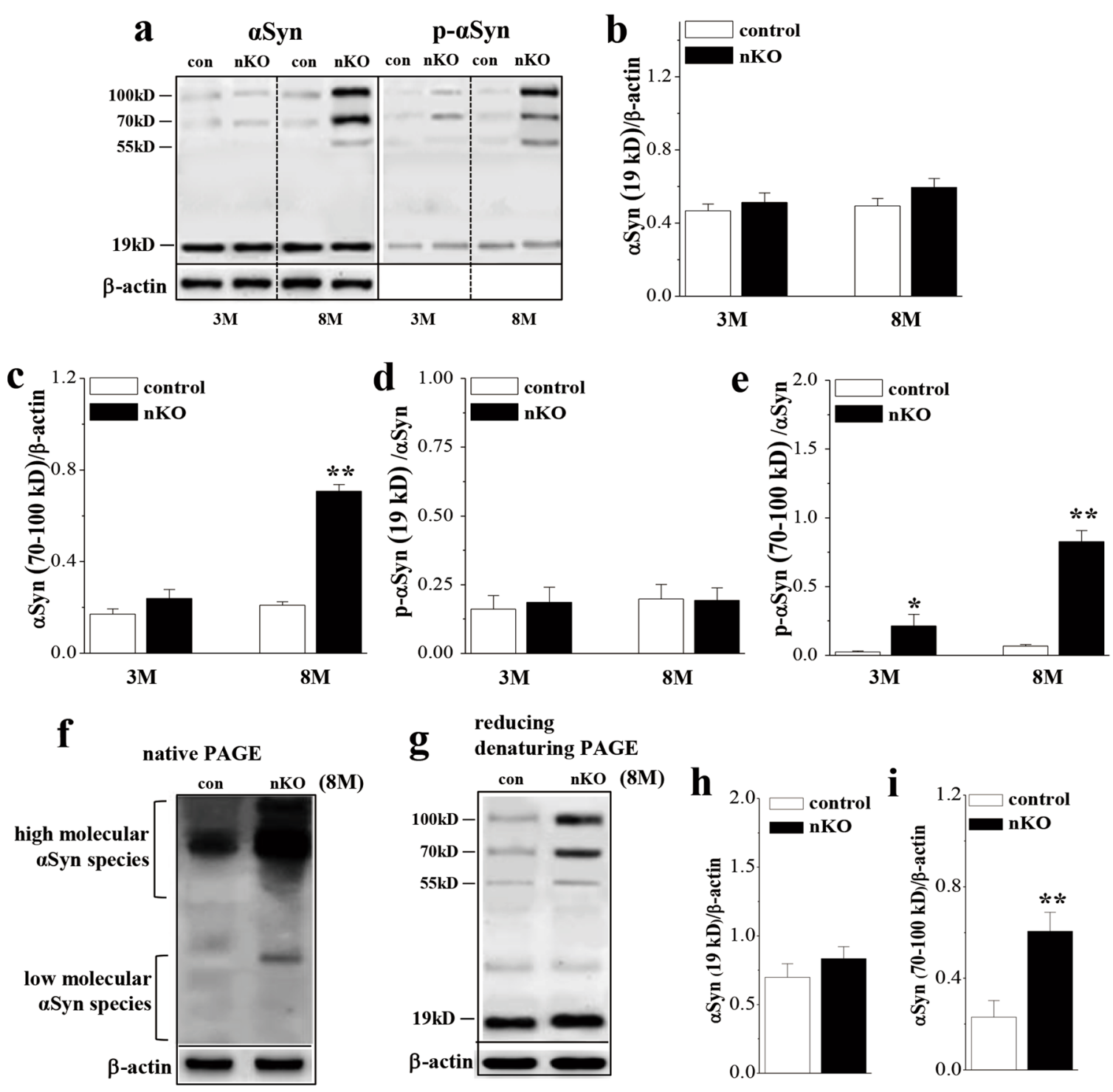

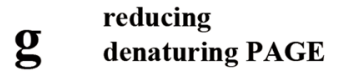
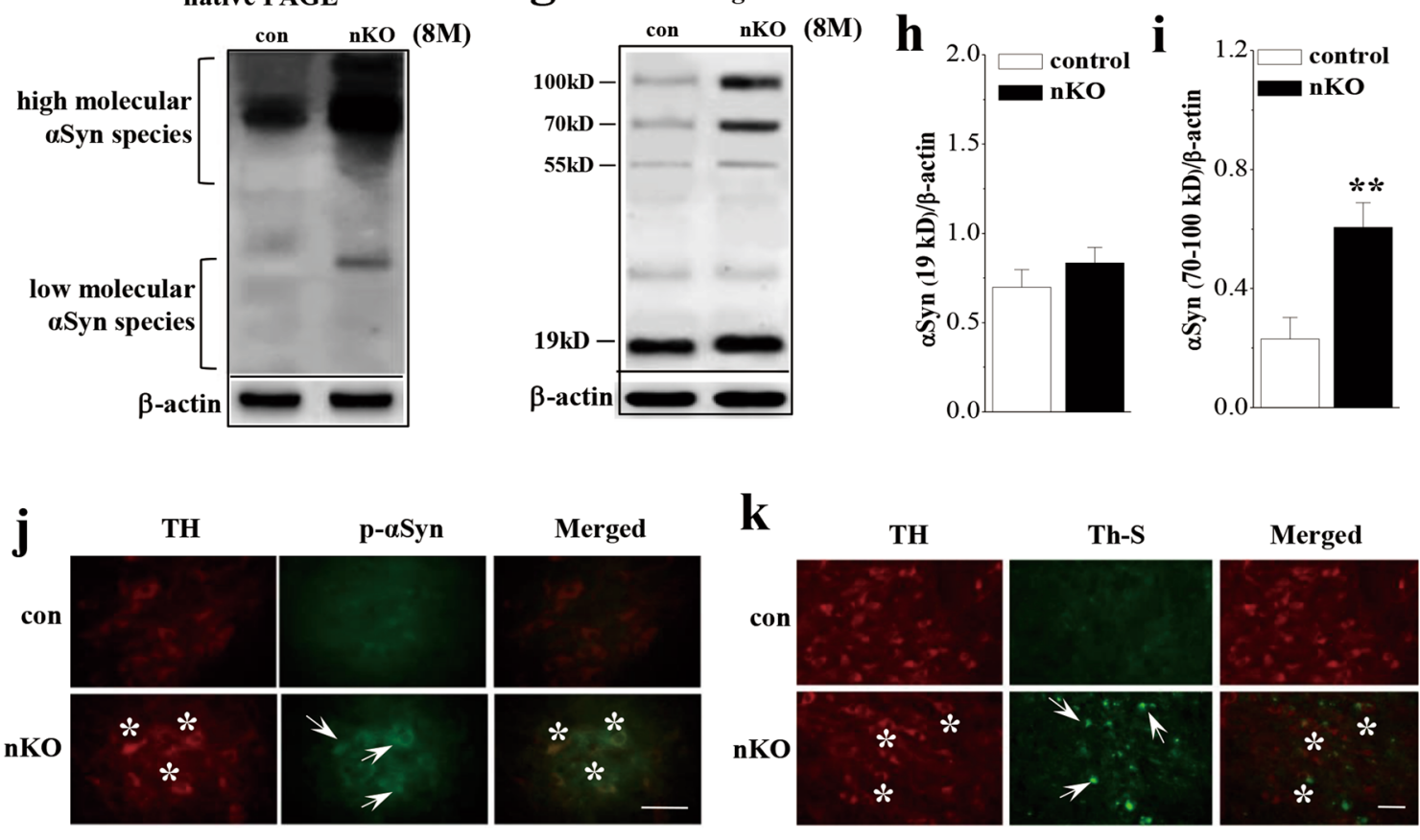

8M

$8 \mathrm{M}$

Fig. 3 Neuronal seipin deficiency increases the accumulation or phosphorylation of aSyn. a Representative immunoblots of aSyn and phosphor-aSyn in SN of 3-M-old and 8-M-old control and seipin-nKO mice. The data of $\beta$-actin in aSyn and phosphor-aSyn are the same. $\mathbf{b}-\mathbf{e}$ The densitometric values for monomers $\left(19 \mathrm{kD}\right.$ ) and oligomers of total and phosphorylation of aSyn ( $p$-aSyn). ${ }^{*} P<0.05$ and ${ }^{* *} P<0.01$ vs. control mice (two-way ANOVA). $\mathbf{f}$ Representative immunoblots of aSyn in a native PAGE in 8-M-old control (con) and seipin-nKO (nKO) mice. g-i Bar graphs show the densitometric values of aSyn monomers and oligomers using reducing denaturing PAGE. ${ }^{*} P<0.01 \mathrm{vs}$. control mice ( $t$-test). j, $\mathbf{k}$ Double immunostaining photographs of phosphor-aSyn (p-aSyn, green) and TH (red) or Th S (green) and TH (red) in SNpc of 8-M-old control and seipin-nKO mice. The arrows indicate p-aSyn or Th S-positive cells; the asterisks represent TH-positive cells. Scale bars $=50 \mu \mathrm{m}(\mathbf{j})$; scale bars $=50 \mu \mathrm{m}(\mathbf{k})$ 


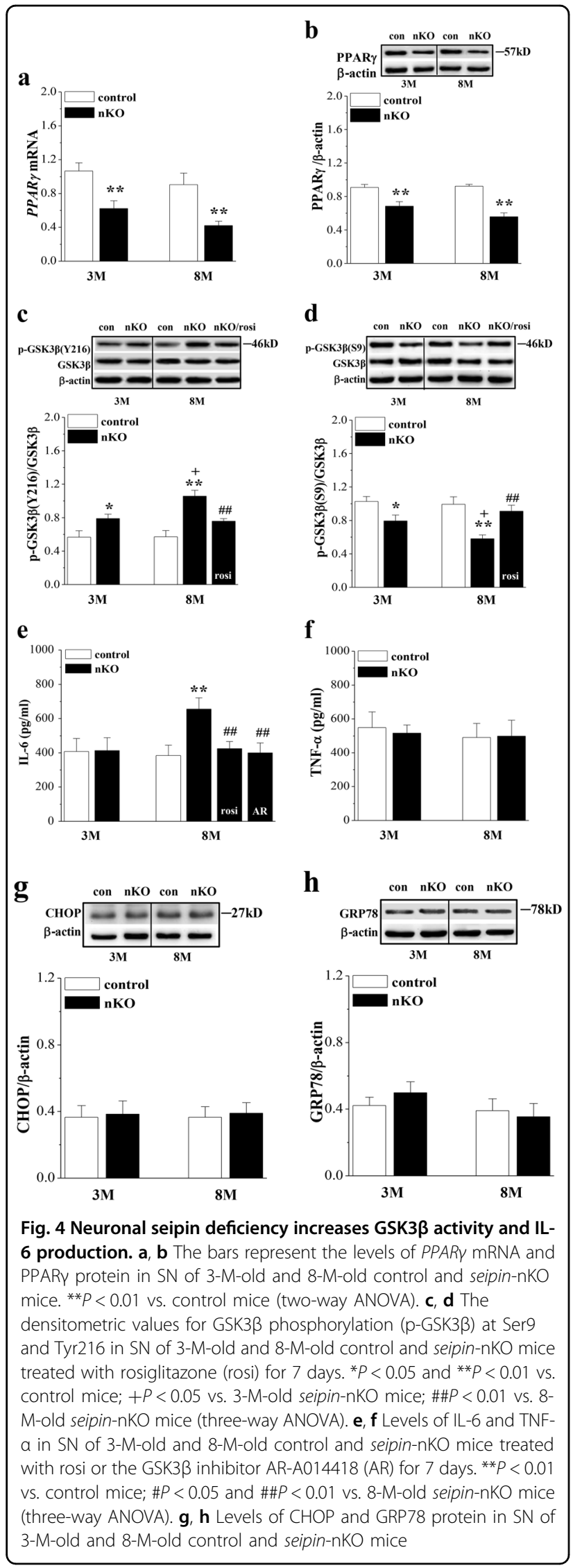

staining of $\mathrm{TH}$ and thioflavin $\mathrm{S}$, a dye that binds to amyloid fibrils ${ }^{22}$, we observed that the TH-positive cells in the 8-M-old seipin-nKO mice was highly overlapped by the thioflavin S-positive structures (Fig. 3k), whereas the thioflavin S-positive structures were barely found in control mice. These results indicate that the seipin deficiency enhances an age-related accumulation and phosphorylation of $\alpha$ Syn, and $\alpha$ Syn fibril formation in dopaminergic neurons.

\section{Influence of seipin deficiency reduced PPARy on neuroinflammation in SN}

Consistent with the previous reports ${ }^{11,20}$, the levels of PPAR $\gamma$ mRNA $(P<0.01, n=6$; Fig. $4 \mathrm{a})$ and PPARy protein $(P<0.01, n=6$; Fig. $4 \mathrm{~b})$ in the SN of 3 -M-old and 8$\mathrm{M}$-old seipin-nKO mice were reduced compared to control mice.

Seipin deficiency in astrocytes has been reported to increase GSK3 $\beta$ activity and levels of pro-inflammatory cytokines through reducing PPAR $\gamma^{15}$. GSK3 $\beta$ activity is negatively and positively regulated by phosphorylation (phospho-GSK3 $\beta$ ) at Ser9 and Tyr216, respectively. There was a main effect of seipin deficiency on phospho-GSK3 $\beta$ at Tyr216 $\left(F_{(1,20)}=25.970, P<0.001\right.$; Fig. $\left.4 \mathrm{c}\right)$ and Ser9 $\left(F_{(1,20)}=22.151, P<0.001\right.$; Fig. $\left.4 \mathrm{~d}\right)$. The level of Tyr216 phospho-GSK3 $\beta$ was elevated $(3 \mathrm{M}: P<0.05 ; 8 \mathrm{M}: P<$ $0.01)$, and the level of Ser9 phospho-GSK3 $\beta$ was reduced (3 M: $P<0.05 ; 8 \mathrm{M}: P<0.01$ ) in 3 -M-old and 8 -M-old seipin-nKO mice compared to those in the controls. Notably, the level of Tyr216 phospho-GSK3 $\beta$ showed an age-related increase in seipin-nKO mice $(P<0.05)$. Importantly, the treatment of 8-M-old seipin-nKO mice with the PPAR $\gamma$ agonist rosiglitazone (rosi, $5 \mathrm{mg} / \mathrm{kg}$ ) for 7 days obviously corrected the elevation of Tyr216 phospho-GSK3 $\beta \quad(P<0.01)$ and the reduction of Ser9 phospho-GSK3 $\beta(P<0.01)$. The level of IL-6 in the SN was affected by genotype $\left(F_{(1,28)}=29.253, P<0.001\right.$; Fig. 4 e) and genotype $\times$ age $\left(F_{(1,28)}=29.253, P<0.001\right)$. The elevation of IL-6 in 8-M-old seipin-nKO mice was sensitive to the 7 days administration of rosi $(P<0.01, n$ $=10)$ or the GSK3 $\beta$ inhibitor AR-A014418 (AR, $1 \mathrm{mg} / \mathrm{kg}$, $P<0.01, n=10)$. By contrast, the level of TNF- $\alpha$ in the SN of 3-M-old and 8-M-old seipin-nKO mice did not significantly differ from control mice $(P>0.05, n=10$; Fig. 4f).

Mutations in seipin can induce ER stress ${ }^{6}$. However, the levels of CHOP (C/EBP homologous protein, transcribed following PERK activation) $(P>0.05, n=6$; Fig. 4 g) or GRP78 protein $(P>0.05, n=6$; Fig. 4 h $)$ in the SN were not significantly different between $3-\mathrm{M}$ old or 8-M-old control mice and seipin-nKO mice. These results indicate that seipin deficiency, through reducing PPAR $\gamma$, enhances age-dependent GSK3 $\beta$ activation, leading to neuroinflammation. 

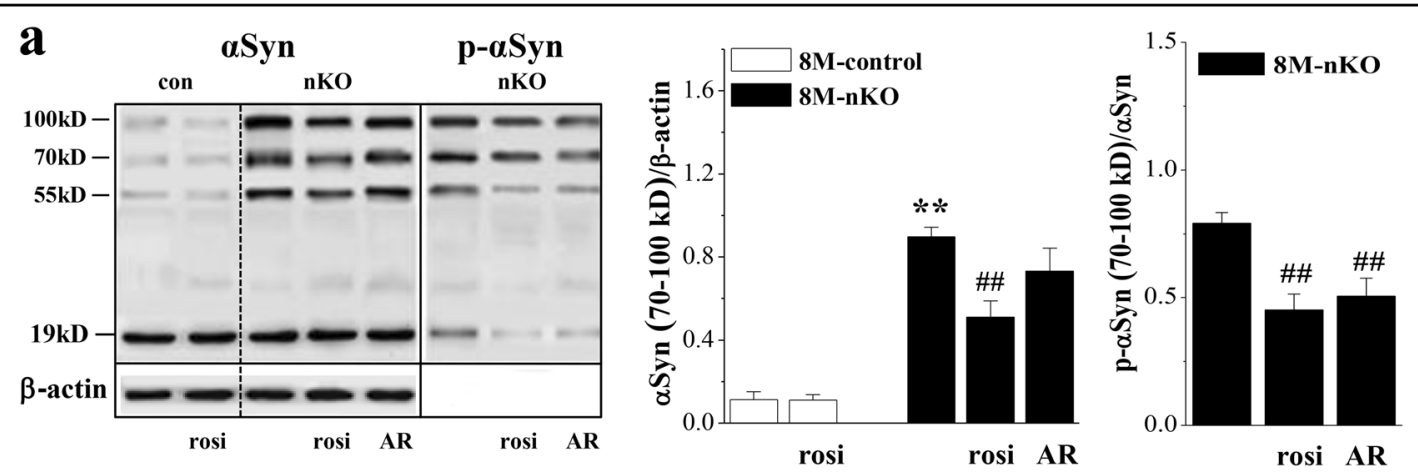

b

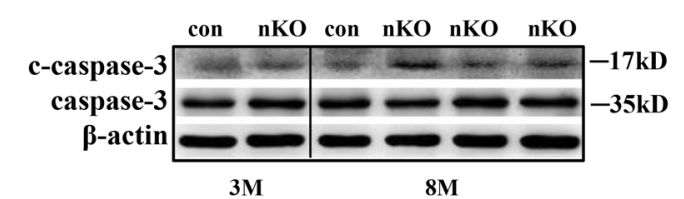

c
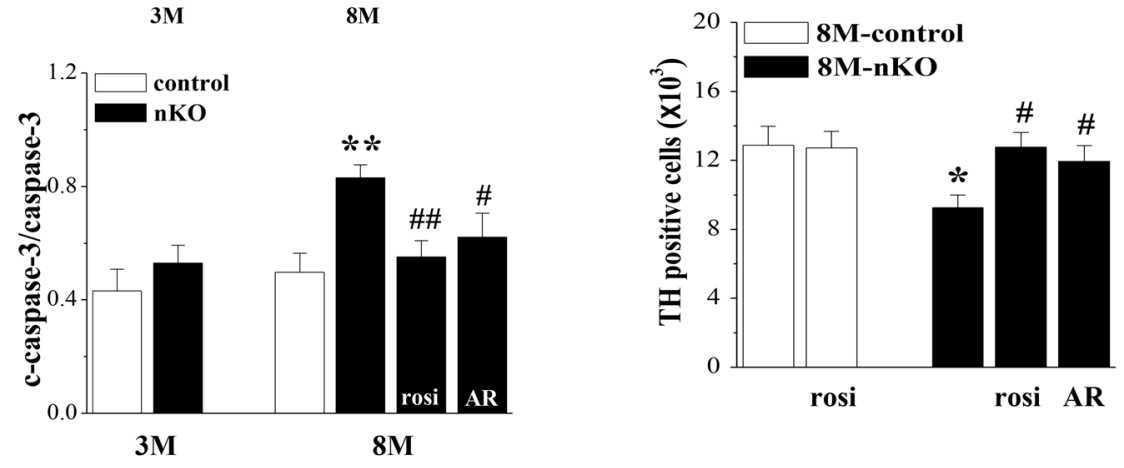

\section{d BWT}

e

RT-AS
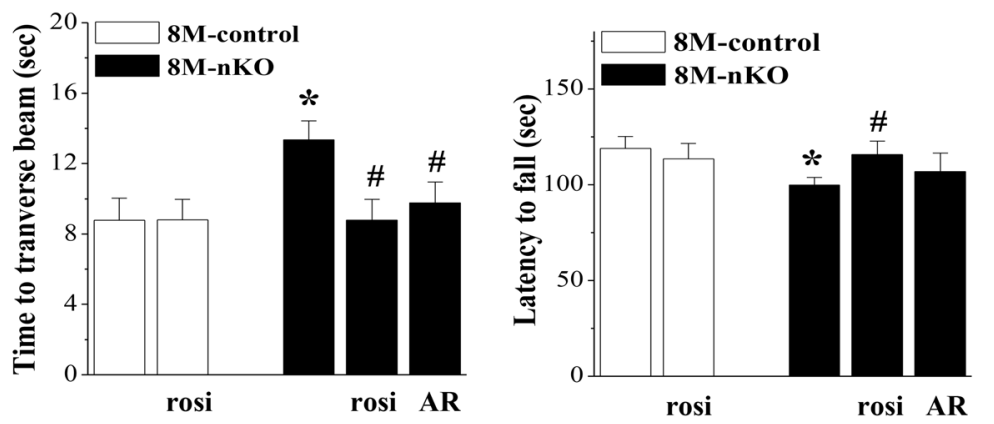

f RT-CS

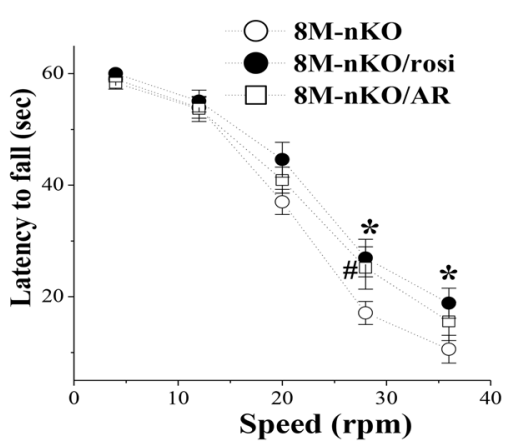

Fig. 5 Effects of rosiglitazone and AR-A014418 on accumulation and phosphorylation of aSyn and impairment of dopaminergic neurons and motor coordination. a Bar graph shows the densitometric values of aSyn oligomers and phosphorylation of aSyn oligomers ( $p$-aSyn) in SN of 8-M-old control and seipin-nKO mice treated with rosi or AR-A014418 (AR) for 28 days. ${ }^{* *} P<0.01$ vs. control mice; \#\#P<0.01 vs. seipin-nKO mice (twoway ANOVA). The data of $\beta$-actin in aSyn oligomers and phosphorylation of aSyn oligomers are the same. $\mathbf{b}$ Level of cleaved caspase-3 (c-caspase-3) in SN of 3-M-old and 8-M-old control mice and seipin-nKO mice treated with rosi or AR. ${ }^{* *} P<0.01$ vs. control mice; \#P<0.05 and \#\#P<0.01 vs. 8-Mold seipin-nKO mice (three-way ANOVA). c Bar graph indicates the number of TH-positive cells in SNpc of 8-M-old control mice and seipin-nKO mice treated with rosi or AR. ${ }^{*} P<0.05$ vs. control mice; $\# P<0.05$ vs. seipin-nKO mice (two-way ANOVA). $\mathbf{d}$, e Bar graphs show the time (s) to traverse the beam in BWT and the latency (s) in RT-AS. ${ }^{*} P<0.05$ vs. control mice; $\# P<0.05$ vs. seipin-nKO mice (two-way ANOVA). $\mathbf{f}$ Each point represents the latency in RT-CS. ${ }^{*} P<0.05$ (seipin-nKO mice treated with rosi) and $\# P<0.05$ (seipin-nKO mice treated with AR) vs. seipin-nKO mice (repeated measures ANOVA) 


\section{Involvement of aSyn in loss of dopaminergic neurons and impaired motor coordination}

To determine the involvement of reduced PPARY and elevated activity of GSK3 $\beta$ in the oligomerization and phosphorylation of $\alpha$ Syn, 7-M-old seipin-nKO mice were treated with rosi $(5 \mathrm{mg} / \mathrm{kg})$ or AR $(1 \mathrm{mg} / \mathrm{kg})$ for 28 days. The treatment of 8-M-old seipin-nKO mice with rosi could correct the increase in the levels of $\alpha$ Syn oligomers $(P<0.01, n=6$; Fig. 5a) and phosphorylation of $\alpha$ Syn $(P<$ $0.01, n=6)$. Similarly, the AR administration in 8-M-old seipin-nKO mice could attenuate the phosphorylation of $\alpha$ Syn $(P<0.01, n=6)$, but it had no effect on the oligomerization of $\alpha$ Syn $(P>0.05, n=6)$.

Finally, we examined the effects of rosi $(5 \mathrm{mg} / \mathrm{kg})$ or AR $(1 \mathrm{mg} / \mathrm{kg})$ on the loss of dopaminergic neurons and impairment of motor coordination in 8-M-old seipin$\mathrm{nKO}$ mice. As shown in Fig. 5b, the level of cleaved caspase-3 (c-caspase-3) in the SN of 8-M-old seipin-nKO mice was higher than that in control mice $(P<0.01, n=$ $6)$, whereas there was no significant difference between 3M-old control mice and seipin-nKO mice $(P>0.05, n=$ 6). The treatment of seipin-nKO mice with rosi perfectly prevented the increase in the level of c-caspase-3 $(P<$ $0.01, n=6)$ and the loss of dopaminergic neurons $(P<$ $0.05, n=6$; Fig. $5 \mathrm{c})$. Furthermore, the deficits in motor coordination of 8-M-old seipin-nKO mice were significantly relieved by the administration of rosi (BWT: $P<$ $0.05, n=12$; Fig. 5d, RT-AS: $P<0.05, n=12$; Fig. 5e, RTCS: $P<0.05, n=12$; Fig. 5 f). The administration of rosi alone in control mice did not cause changes in motor coordination (BWT: $P>0.05, n=12$; RT-AS: $P>0.05, n$ $=12$ ). In contrast, the inhibited GSK3 $\beta$ activation by AR partially reduced the level of c-caspase-3 $(P<0.05, n=6)$ and the death of dopaminergic neurons $(P<0.05, n=6)$, and could alleviate the deficits in motor coordination (BWT: $P<0.05, n=12$; RT-CS: $P<0.05, n=12$ ) in 8 -Mold seipin-nKO mice.

\section{Discussion}

Using the seipin-sKO mice, seipin-nKO mice, and seipin-aKO mice models, the present study provides evidence that seipin deficiency in dopaminergic neurons through reducing PPAR $\gamma$ expression enhances the aggregation and phosphorylation of $\alpha$ Syn and induces the neuroinflammatory, which leads to the loss of dopaminergic neurons and the deficits of motor coordination. This conclusion is deduced mainly from the following observations. First, 8-M-old and 12-M-old seipin-nKO mice and 8-M-old seipin-sKO mice exhibited decline in motor coordination, but the seipin-aKO mice did not. Therefore, it is conceivable that the deficiency in motor coordination is induced by the neuronal seipin deficiency, which is unlikely to be secondary to the peripheral metabolic abnormalities. In addition, the neuronal seipin deficiency does not affect motor ability, as the phenotypes of motor neurons impaired by the gain-of-function mutation in the $\mathrm{N}$-glycosylation site of seipin ${ }^{6}$ are not observed in seipin-sKO, seipin-nKO, and seipin-aKO mice $^{11}$ in the present study or in seipin knockout rats ${ }^{23}$. Second, the dopaminergic neurons in the SNpc highly expressed the seipin protein. The number of TH-positive cells was reduced by approximately 28 and $43 \%$ in 8 -Mold and 12-M-old seipin-nKO mice. Third, the levels of $\alpha$ Syn oligomer, phosphor- $\alpha$ Syn, and IL-6 in the SN of 8$\mathrm{M}$-old seipin-nKO mice were significantly increased. Moreover, the seipin deficiency increased the $\alpha$ Syn fibril formation in dopaminergic neurons. Fourth, the activation of PPAR $\gamma$ by rosiglitazone in 8-M-old seipin-nKO mice could reduce the aggregation and phosphorylation of $\alpha$ Syn and correct the elevation of IL- 6 , and prevent the loss of dopaminergic neurons, which was accompanied by the recovery of motor coordination.

\section{Seipin deficiency enhances the oligomerization of aSyn by reducing PPARY}

The level of PPARy in the SN of seipin-nKO mice was reduced, and the PPAR $\gamma$ activator could prevent $\alpha$ Syn oligomerization. It is highly likely that the reduction of PPAR $\gamma$ enhances the $\alpha$ Syn oligomerization. The idea is supported by a recent study ${ }^{24}$ that PPAR $\gamma$, as a binding partner for transcription factor retinoic $\mathrm{X}$ receptor, prevents the formation of $\alpha$ Syn oligomers. Growing evidence suggests that polyunsaturated fatty acids can promote $\alpha$ Syn oligomerization and aggregation ${ }^{25}$. The exposure of dopaminergic neurons to physiological polyunsaturated fatty acids concentrations can elevate the levels of soluble $\alpha$ Syn oligomers and insoluble $\alpha$ Syn aggregates ${ }^{26}$ and increases the deposition of $\alpha$ Syn into cytoplasmic intraneuronal Lewy-like inclusions ${ }^{21}$. The seipin deficiency has been reported to induce the accumulation of phosphatidic acids in neuronal cells, which is able to suppress the PPARY expression ${ }^{20}$. Thus, one possible explanation is that the seipin deficiency via the accumulation of phosphatidic acids leads to the decline of PPAR $\gamma$ concentration, which enhances the $\alpha$ Syn oligomerization. In addition, the $\alpha$ Syn phosphorylation, especially at serine 129 , has been shown to promote $\alpha$ Syn aggregation ${ }^{27}$. The activation of GSK-3 $\beta$ has been reported to affect $\alpha$ Syn protein expression and oligomerization ${ }^{17,18}$. A recent study has reported that the GSK- $3 \beta$ inhibitors can decrease the $\alpha$ Syn accumulation ${ }^{28}$. However, the inhibition of GSK-3 $\beta$ by AR-A014418 failed to alter the oligomerization of $\alpha$ Syn in seipin-nKO mice, although it reduced the phosphorylation of $\alpha$ Syn. 


\section{Seipin deficiency enhances aSyn phosphorylation through GSK-3 $\beta$ activation}

Serine 129 phosphorylation is one of the most important post-translational modifications of $\alpha$ Syn. The phosphorylated S129 $\alpha$ Syn is co-localized with phosphorylated Tyr216 GSK-3 $\beta$. And, the activation of GSK-3 $\beta$ can directly phosphorylate $\alpha$ Syn at a single site, $S 129^{29}$. In the SN of seipin-nKO mice, the catalytic activity of GSK3 $\beta$ was enhanced as indicated by the elevation of Tyr 216 phospho-GSK3 $\beta$ and the reduction of Ser9 phosphoGSK3 $\beta$ without the changes in the level of GSK3 $\beta$ protein, which could be corrected by the PPAR $\gamma$ agonist. Accumulating evidence supports the notion that GSK3 $\beta$ is a strong promoter of pro-inflammatory cytokines, including IL- 6 and TNF- $\alpha$. The level of IL- 6 was elevated in the SN of seipin-nKO mice, whereas the level of TNF- $\alpha$ was not altered significantly. TNF- $\alpha$ is mainly released by activated microglia. The seipin protein is expressed in astrocytes, not in microglia ${ }^{15}$. Thus, the seipin deficiency in astrocytes increases the production of IL- 6 via the activation of GSK3 $\beta$. The elevation of IL-6 and phosphorylation of $\alpha$ Syn in 8-M-old seipin-nKO mice were sensitive to the GSK3 $\beta$ inhibitor and the PPARy agonist, but it remains unclear whether $\alpha$ Syn phosphorylation is consequence of inflammation. On the other hand, ER stress is known to promote $\alpha$ Syn phosphorylation ${ }^{30}$. The $\alpha$ Syn phosphorylation can induce the activation of ER stress ${ }^{31}$. Consistent with the results observed in the hippocampus ${ }^{11}$, the levels of the ER stress markers GRP78 and CHOP were not increased in the SN of seipin-nKO mice.

\section{Seipin deficiency leads to an age-related loss of dopaminergic neurons}

Another critical finding in the present study is that seipin-nKO mice showed an age-related loss of dopaminergic neurons and deficits in motor coordination. The administration of the PPAR $\gamma$ agonist or the GSK3 $\beta$ inhibitor in seipin-nKO mice could reduce the death of dopaminergic neurons and improve the deficits in motor coordination, although the PPAR $\gamma$-mediated effects seem to be larger than those of the GSK3 $\beta$ inhibitor. The reduced PPAR $\gamma$ expression or the activated phosphorylation of GSK-3 $\beta$ was detected in young seipin-nKO mice, while the accumulation and phosphorylation of $\alpha$ Syn or the level of IL- 6 were increased in an agedependent manner. Aging itself is accompanied by increased accumulation of phosphorylated $\alpha$ Syn oligomers in monkeys ${ }^{32}$. Aging is associated with changes in the motor coordination ${ }^{33}$. Additionally, the neuroinflammatory process in Parkinson's disease brain is possibly triggered by aging ${ }^{34}$. The PPAR $\gamma$-knockout in mice does not cause the death of neuronal cells, but it can increase the susceptibility to brain damage after cerebral ischemia $^{35}$. The small $\alpha$ Syn aggregates (oligomers and

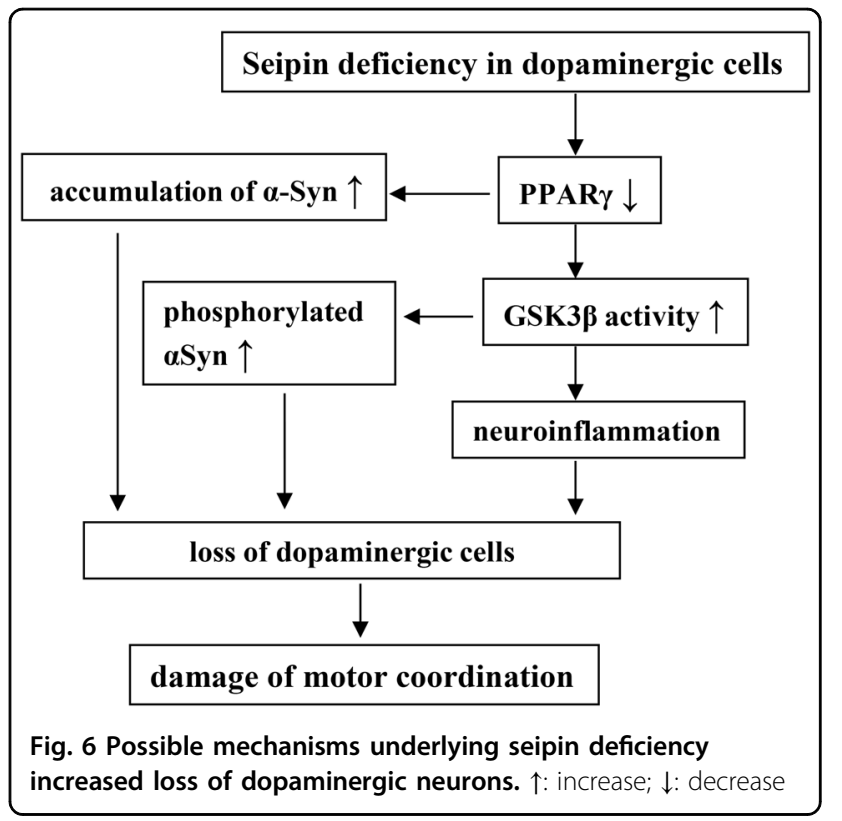

protofibrils) rather than the $\alpha$ Syn fibrils are reported to be toxic to neurons ${ }^{14,36}$. A progressive conversion of the soluble $\alpha$ Syn protein into insoluble, $\beta$-sheet-rich filaments, and their intraneuronal deposition into Lewy bodies underlie its cytotoxicity in the synucleinopathies ${ }^{37}$. The phosphorylated $\alpha$ Syn oligomers have a potential neurotoxic effect, although at the lower levels it may not induce obvious neurodegeneration. The accumulation of $\alpha$ Syn may increase the vulnerability of dopaminergic neurons to inflammation, as genetic ablation of $\alpha$ Syn decreases the sensitivity of cells to inflammatory challenges ${ }^{38}$. Taken together with the discussion, the seipin deficiency through reducing PPAR $\gamma$ causes the agerelated loss of dopaminergic neurons probably by at least two possible mechanisms: one is increased oligomerization of $\alpha$ Syn, and the other is enhanced neuroinflammation and phosphorylation of $\alpha$ Syn via activation of GSK3 $\beta$ (Fig. 6).

\section{Conclusion}

The seipin deficiency through enhanced aggregation and phosphorylation of $\alpha$ Syn or neuroinflammation causes the loss of dopaminergic neurons leading to deficits in motor coordination. Overall, the results presented herein can help for understanding underlying pathogenic processes and mechanisms of Parkinson's disease.

\section{Materials and methods}

Experimental animals

All experiments were carried out in accordance with the guidelines established by the Institute for Laboratory Animal Research of Nanjing Medical University. All animals were housed in a light-controlled room under a $12-\mathrm{h}$ 
light-dark cycle (starting at 07:00 h) and were maintained at $23^{\circ} \mathrm{C}$ in the Animal Research Center of Nanjing Medical University. The animals had free access to food and water before and after all procedures. The seipinsKO, seipin-nKO, and seipin-aKO mice were generated as previously described ${ }^{11,19}$. Briefly, neuron-specific and adipose-specific deletion of seipin exon 3 was induced by crossing mice with the loxP seipin allele with transgenic mice expressing Cre recombinase driven by a neuronspecific or an adipocyte-specific promoter (nestin-Cre or aP2-Cre; Jackson Laboratories). Progenies were screened by PCR for loss of the seipin exon 3 and presence of nestin-Cre or aP2-Cre. The genotype of seipin-nKO and seipin-aKO mice was identified by PCR using genomic DNA from their tails. When heterozygous seipin LoxPnestin or aP2-Cre were obtained, they were further crossed with seipin loxP/loxP homozygous mice. The progenies were screened by genomic DNA PCR for seipin $\operatorname{lox} \mathrm{P} /$ loxP with nestin-Cre or aP2-Cre, which would be used in subsequent experiments. Progenies were also screened by PCR for loss of the seipin exon 3 and presence of nestin-Cre, which would be used in subsequent experiments. The DNA was amplified with the following primers: 5'-CTTGTCTCAAAGGGGTCT-3' (forward primer for loxP) and 5'-TCAACAGAACAGACGCT-3' (reverse primer for loxP); and 5'-GCGGTCTGGCAGTAAAAACTATC-3' (forward primer for nestin-Cre) and 5'-GTGAAACAGCATTGCTGTCACTT-3' (reverse primer for nestin-Cre).

\section{Experimental design}

Male 3-M-old $(n=22), 8$-M-old $(n=66)$, and 12-M-old $(n=12)$ seipin- $\mathrm{nKO}$ mice, 8 -M-old seipin-sKO mice $(n=$ $12)$ or 8-M-old seipin-aKO mice $(n=12)$ and agematched control mice (nestin-Cre mice) $(n=12)$ or WT mice $(n=62)$ were used at the beginning of all experiments and divided into five experimental groups: the first group was used to examine spontaneous activity and motor coordination, number of dopaminergic neurons, levels of $\alpha$ Syn monomers, $\alpha$ Syn oligomers, and oligomer phosphorylation; the second group was used to measure the levels of cleaved caspase-3, PPAR $\gamma$ expression, ER stressers, phosphorylation of GSK3 $\beta$, IL- 6 , and TNF- $\alpha$; the third group was examined the phosphorylation of $\alpha$ Syn in dopaminergic neurons; the fourth and fifth groups were treated with rosiglitazone or AR-A014418. At the end of the behavioral tests the mice were perfusion-fixed for histological examination or were decapitated for biochemical assays and western blotting.

\section{Behavioral examination}

Three different behavioral tests were carried out (9000-1400) under the following sequence: open-field test $\rightarrow$ beam walking test $\rightarrow$ rotarod test. The tests were spaced by $24 \mathrm{~h}$. These behavioral tests were recorded by a video monitor (Winfast PVR; Leadtek Research Inc., Fremont, CA). The results of the OFT and BWT were analyzed using TopScan Lite 2.0 (Clever Sys, Reston, VA), and the results of the RT were analyzed by Rota-Rod Microprocessor 47600 (Ugo Basile, Biological Research Apparatus, Varese, Italy). Open-field test: Each mouse was placed in a clear, open-top, square Plexiglas box $(30 \mathrm{~cm} \times$ $30 \mathrm{~cm} \times 40 \mathrm{~cm}$ ) in a subdued room and allowed to freely explore for $6 \mathrm{~min}$. Total traveled distance was measured within $6 \mathrm{~min}$. Beam walking test: The challenging beam was a $1-\mathrm{m}$ long wooden beam suspended $23 \mathrm{~cm}$ above a bench top covered with soft pads. The beam was divided in four gradually narrowing sections $(25 \mathrm{~cm} /$ section $)$ at widths of $3.5,2.5,1.5$, and $0.5 \mathrm{~cm}$. The beam was covered with surgical tape that provided sufficient surface traction for the animals to walk on. All mice were pre-trained on traversing the beam for 2 consecutive days. On the third day, each mouse tested with five trials (inter-trial intervals $=10-12 \mathrm{~s}$ ), and the average time was calculated ${ }^{39}$. Rotarod test: Each mouse was placed in the forward position on a rotating rod (rotarod apparatus, Ugo Basile, Varese, Italy). On days 1 and 2, the mice learned to stay on the rotarod at a constant speed $(20 \mathrm{rpm})$ for $300 \mathrm{~s}$. For a RT with constant speeds (RT-CS), motor coordination was assessed with five constant speeds (4-36 rpm) for a maximum of $60 \mathrm{~s}$ at each speed on day 3. For a RT with accelerating speed (RT-AS), motor coordination was assessed with an accelerating speed (4-45 rpm) over a period of $2.5 \mathrm{~min}$. The mice were tested twice at each speed with a resting period of $20 \mathrm{~min}$. For each trial, the time (latency) until the mice fall off the rotarod was recorded.

\section{Histological examination}

Mice were anesthetized with chloral hydrate $(400 \mathrm{mg} /$ $\mathrm{kg}$, i.p.) and then perfused with $4 \%$ paraformaldehyde. The brains were transferred into $30 \%$ sucrose. TH immunohistochemistry and quantitative analyses: coronal sections $(30 \mu \mathrm{m})$ were cut using a cryostat after gradient dehydration. The sections were treated with $5 \%$ normal goat serum and then incubated in chicken anti-TH (1:1000; Abcam ab76442, Cambridge, UK) at $4{ }^{\circ} \mathrm{C}$ overnight. The sections were incubated in biotin-labeled goat antichicken IgG antibody (1:500; Abcam ab6876) for $2 \mathrm{~h}$. Immunoreactivities were visualized by avidin-biotin horseradish peroxidase complex (ABC Elite; Vector Laboratories, Inc., Burlingame, CA, USA) using 3,3'-diaminobenzidine as a chromogen. The sections were observed using a microscope (Olympus DP70, Tokyo, Japan) with a $40 \times$ objective. Every third section (12 sections per mouse) was obtained for consecutive cell quantification analyses. Stereological cell counting was performed with a stereological system consisting of a light 
microscope with a CCD camera (Olympus DP70), a motorized specimen stage for automatic sampling (MicroBrightField, Williston, VT, USA) and a computer running MicroBrightField Stereo Investigator software (MicroBrightField $)^{40}$. The section thickness was measured using a dissector height of $5 \mu \mathrm{m}$. The total number of THpositive cells was estimated using the optical fractionator formula: number of neurons $=1 /$ ssf (slice sampling fraction) $\times 1 /$ asf (area sampling fraction) $\times 1 /$ tsf (thickness sampling fraction) $\times \Sigma$ (number of objects counted). TH/ seipin or $T H / p-\alpha S y n$ dual-antigen immunofluorescence: The sections $(30 \mu \mathrm{m})$ were treated with $5 \%$ normal goat serum and then incubated in a chicken anti-TH antibody (1:1000; Abcam ab76442) and rabbit anti-seipin antibody (1:1000; Abcam ab106793) ${ }^{41}$ or rabbit anti-phosphor$\alpha$ Syn antibody (1:1000; Abcam ab51253), at $4{ }^{\circ} \mathrm{C}$ overnight. The seipin monoclonal antibody was a kind gift from Professor Jiahao Sha (State Key Laboratory of Reproductive Medicine, Nanjing Medical University, China). The sections were incubated in Alexa Fluor 594conjugated AffiniPure donkey anti-chicken antibody (1:200, Jackson ImmumoResearch, West Grove, PA), Alexa Fluor 488-conjugated Affinipure donkey anti-rabbit antibody (1:200, Jackson ImmumoResearch) for $2 \mathrm{~h}$. Sections were coverslipped with Antifade Mounting Medium (Sigma-Aldrich) and viewed under a fluorescent microscope (Olympus DP70) with a 100× objective. Double immuno-staining of TH/Thioflavin S: The $\alpha$ Syn fibril formation was monitored by Th-S binding. Th-S is a fluorescent dye that interacts with fibrils in a $\beta$-sheet structure. The sections $(30 \mu \mathrm{m})$ were pre-incubated with $5 \%$ normal horse serum and then incubated in a chicken anti- $\mathrm{TH}$ antibody at $4{ }^{\circ} \mathrm{C}$ overnight. After phosphatebuffered saline (PBS) rinses, the sections were incubated Alexa Fluor 594-conjugated AffiniPure donkey antichicken antibody (1:200, Jackson ImmumoResearch) for $2 \mathrm{~h}$. Then, the sections were incubated in Thioflavin S (10 $\mu \mathrm{g} / \mathrm{ml}$; Sigma-Aldrich) for $5 \mathrm{~min}$. After PBS washing, sections were coverslipped with Antifade Mounting Medium (Sigma-Aldrich) and viewed using a fluorescent microscope (Olympus DP70) with a 100x objective.

\section{Western blot analysis}

The brains were removed quickly under anesthesia with isoflurane. The coronal sections $(500 \mu \mathrm{m})$ of midbrain were cut using a cryostat. The regions of containing SN were harvested using a 15-gauge needle (inner diameter $=1.5 \mathrm{~mm}$ ). The samples were homogenized in lysis buffer (50 mM Tris- $\mathrm{HCl}, 150 \mathrm{mM} \mathrm{NaCl}, 5 \mathrm{mM}$ EDTA, $10 \mathrm{mM}$ NaF, $1 \mathrm{mM}$ sodium orthovanadate, $1 \%$ TritonX-100, $0.5 \%$ sodium deoxycholate, $1 \mathrm{mM}$ phenylmethylsulfonyl fluoride, and protease inhibitor cocktail) (Roche, Germany). The protein concentration was measured using a BCA protein assay kit (Pierce, IL, USA). For SDS-PAGE experiments, proteins were suspended in non-reducing loading buffer $(0.3 \mathrm{M}$ Tris- $\mathrm{HCl}, 5 \%$ SDS, and $50 \%$ glycerol) or reducing loading buffer $(0.3 \mathrm{M}$ Tris- $\mathrm{HCl}, 5 \%$ SDS, 50\% glycerol, and $100 \mathrm{mM}$ dithiothreitol) (Pierce), and then boiled. For native-PAGE experiment, the samples were homogenized in solubilization buffer containing $50 \mathrm{mM}$ BisTris- $\mathrm{HCl}$ (pH 7.0), $500 \mathrm{mM}$ 6-amino-caproic acid, $10 \%$ glycerol, $1 \%$ digitonin (20\% stock in water), cocktail and phosphatase inhibitor, and then suspended in loading buffer (5\% Serva G250, $50 \mathrm{mM}$ BisTris-HCl, 500 $\mathrm{mM}$ 6-amino-caproic acid) without boiling directly onto gels $^{42}$.

After transfer of proteins to polyvinylidene difluoride membranes (Millipore), the membranes were incubated with $5 \%$ nonfat dried milk for $60 \mathrm{~min}$ and then incubated with rabbit anti-seipin (1:1000; Abcam ab106793), rabbit anti-phosphorylated $\alpha$ Syn (1:1000; Abcam ab51253), rabbit anti-phosphorylated GSK3 $\beta$ (S9, 1:1000; Cell Signaling Technology 9336S, Inc., Boston, MA, USA), mouse anti-phosphorylated GSK3 $\beta$ (Y216, 1:1000; BD Transduction Laboratories 612313, Lexington, KY, USA), mouse anti-cleaved caspase-3 (c-caspase-3, 1:1000; Cell Signaling Technology 9661S), rabbit anti-PPARY (1:1000; Santa Cruz sc-7196, Fremont, CA, USA), rabbit antiGRP78 (1:1000; Bioworld Technology BS1154, St. Louis Park, MN, USA), or rabbit anti-CHOP (1:500; Cell Signaling Technology 2895S) at $4{ }^{\circ} \mathrm{C}$ overnight. The membranes were incubated with horseradish peroxidaselabeled secondary antibodies and visualized using an ECL detection kit (Millipore), and images were acquired using the ChmiDOC XRS Imaging System (Bio-Rad Laboratories, CA, USA). Optical density of the immunoreactive bands was measured using NIH ImageJ Software (Bethesda, MD, USA). Following visualization, the blots were stripped by incubation in stripping buffer (Restore, Pierce) for $5 \mathrm{~min}$, re-blocked with $5 \%$ nonfat dried milk for $60 \mathrm{~min}$, and re-incubated with rabbit antiGSK3 $\beta$ (1:1000; Cell Signaling Technology 9315S), rabbit anti-caspase-3 (1:1000; Cell Signaling Technology 9662S), or mouse anti- $\beta$-actin (1:1000; Abbkine A01010, Redlands, CA, USA) or mouse anti- $\alpha$ Syn (anti-synuclein-1, 1:1000; BD Transduction Laboratories 610786, Lexington, KY, USA). The optical density of specific bands was then normalized to the corresponding GSK3 $\beta, \alpha$ Syn, or $\beta$-actin levels.

\section{Treatment with drugs}

The PPAR $\gamma$ agonist rosiglitazone (rosi; Enzo, Farmingdale, NY, USA) and the GSK3 $\beta$ antagonist AR-A014418 (AR; Sigma-Aldrich, MO, USA) were dissolved in dimethyl sulfoxide (DMSO) and then diluted in saline to a final concentration of $0.5 \%$ DMSO. Oral administration (p.o.) of rosi $(5 \mathrm{mg} / \mathrm{kg})^{43}$ or intraperitoneal injection (i.p.) of AR-A014418 $(1 \mathrm{mg} / \mathrm{kg})^{44}$ was given daily. 


\section{Enzyme-linked immunosorbent assay (ELISA)}

The samples of $\mathrm{SN}$ were homogenized in PBS solution and centrifuged at $12,000 \mathrm{rpm}$ for $15 \mathrm{~min}$ at $4{ }^{\circ} \mathrm{C}$, and the supernatant was obtained for further analyses. Sample aliquots of $100 \mu \mathrm{l}$ were used to measure TNF- $\alpha$ and IL-6 using mouse cytokine ELISA kits from R\&D Systems (Minneapolis, MN), according to the manufacturer's instructions. The absorbance for all of the cytokines studied was measured using a SpectraMax Plus 384 Microplate Reader (Molecular Devices, MD, USA) at $450 \mathrm{~nm}$.

\section{Quantitative real-time reverse-transcription PCR}

The samples of the SN were quickly taken, and total RNA was isolated using TRIzol reagent (Invitrogen, Carlsbad, CA, USA). RNA $(1 \mu \mathrm{g})$ was used to reverse transcribe using high-capacity cDNA of the reverse transcription kit RT (TaKaRa Biotechnology Co., Ltd.). The primer sequences of PPARY (forward primer: 5'GCTTATTTATGATAGGTGTGATC-3'; reverse primer: $5^{\prime}$-GCATTGTGAGACATCCCCAC-3') and GAPDH mRNA (forward primer: 5'-ACCACAGTCCATGCCATCAC-3'; reverse primer: 5'-ACCACAGTCCATGCCATCAC-3') were designed according to a previous publication ${ }^{11}$. Q-RT-PCR was performed using a Light Cycler Fast Start DNA Master SYBR Green I kit and an ABI Prism 7300 Sequence Detection System (Applied Biosystems, Foster City, California, USA). The relative expression of genes was determined using the 2$\triangle \Delta$ ct method, with normalization to GAPDH expression. The levels of PPAR $\gamma$ mRNA were expressed as percent of control mice.

\section{Data analysis/statistics}

Data were retrieved and processed with Microcal Origin 8.0. The group data were expressed as the mean \pm standard error. All statistical analyses were performed using SPSS software, version 20.0 (SPSS Inc., USA). Differences among the means were analyzed using the analysis of variance (ANOVA) with or without repeated measures, followed by post hoc Bonferroni's test, where appropriate. Differences at $P<0.05$ were considered to be statistically significant.

\section{Funding}

This study was supported by the National Natural Science Foundation of China (81471157; 81671253), the National 973 Basic Research Program of China (2014CB943303), and Jiangsu provincial Science Foundation (EB2016765).

\footnotetext{
Authors' contributions

L.W. performed the western blot analysis and behavioral examinations. J.H. participated in the histological examinations and all statistical analyses. Y.W. contributed to the animal care. G.L. participated in for the experimental design. L.C. and W.Y. are responsible for the experimental design and finished the manuscript.
}

\begin{abstract}
Author details
${ }^{1}$ State Key Lab of Reproductive Medicine, Nanjing Medical University, 210029 Nanjing, China. ${ }^{2}$ Department of Physiology, Nanjing Medical University, 210029 Nanjing, China. ${ }^{3}$ Institute of Cardiovascular Sciences, Peking University and Key Laboratory of Cardiovascular Sciences, China Administration of Education, 100191 Beijing, China. ${ }^{4}$ Key Lab of Molecular Biology, Guiyang Medical University, 550004 Guiyang, Guizhou, China
\end{abstract}

\section{Conflict of interest}

The authors declare that they have no conflict of interest.

\section{Publisher's note}

Springer Nature remains neutral with regard to jurisdictional claims in published maps and institutional affiliations.

Received: 31 August 2017 Revised: 12 February 2018 Accepted: 9 March 2018

Published online: 18 April 2018

\section{References}

1. Agarwal, A. K. et al. Phenotypic and genetic heterogeneity in congenital generalized lipodystrophy. J. Clin. Endocrinol. Metab. 88, 4840-4847 (2003).

2. Szymanski, K. M. et al. The lipodystrophy protein seipin is found at endoplasmic reticulum lipid droplet junctions and is important for droplet morphology. Proc. Natl Acad. Sci. USA 104, 20890-20895 (2007).

3. Rajab, A., Khaburi, M., Spranger, S., Kunze, J. \& Spranger, J. Congenital generalized lipodystrophy, mental retardation, deafness, short stature, and slender bones: a newly recognized syndrome? Am. J. Med. Genet. A 121A, 271-276 (2003).

4. Ebihara, K. et al. Gene and phenotype analysis of congenital generalized lipodystrophy in Japanese: a novel homozygous nonsense mutation in seipin gene. J. Clin. Endocrinol. Metab. 89, 2360-2364 (2004).

5. Magre, J. et al. Identification of the gene altered in Berardinelli-Seip congenital lipodystrophy on chromosome 11q13. Nat. Genet. 28, 365-370 (2001).

6. Ito, D. \& Suzuki, N. Seipinopathy: a novel endoplasmic reticulum stressassociated disease. Brain 132, 8-15 (2009).

7. Ito, D., Fujisawa, T., lida, H. \& Suzuki, N. Characterization of seipin/BSCL2, a protein associated with spastic paraplegia 17. Neurobiol. Dis. 31, 266-277 (2008).

8. Auer-Grumbach, M. et al. Phenotypes of the N88S Berardinelli-Seip congenital lipodystrophy 2 mutation. Ann. Neurol. 57, 415-424 (2005).

9. Irobi, J. The phenotype of motor neuropathies associated with BSCL2 mutations is broader than Silver syndrome and distal HMN type V. Brain 127, 2124-2130 (2004)

10. Yagi, T., Ito, D., Nihei, Y., Ishihara, T. \& Suzuki, N. N88S seipin mutant transgenic mice develop features of seipinopathy/BSCL2-related motor neuron disease via endoplasmic reticulum stress. Hum. Mol. Genet. 20, 3831-3840 (2011).

11. Zhou, L. et al. Lack of seipin in neurons results in anxiety- and depression-like behaviors via down regulation of PPARgamma. Hum. Mol. Genet. 23, 4094-4102 (2014)

12. Licker, $\mathrm{V}$. et al. Proteomic analysis of human substantia nigra identifies novel candidates involved in Parkinson's disease pathogenesis. Proteomics 14, 784-794 (2014).

13. van Dijk, K. D. et al. The proteome of the locus ceruleus in Parkinson's disease: relevance to pathogenesis. Brain Pathol. 22, 485-498 (2012).

14. Colla, E. et al. Accumulation of toxic a-synuclein oligomer within endoplasmic reticulum occurs in a-synucleinopathy in vivo. J. Neurosci. 32, 3301-3305 (2012).

15. Qian, Y. et al. Neuronal seipin knockout facilitates Abeta-induced neuroinflammation and neurotoxicity via reduction of PPARgamma in hippocampus of mouse. J. Neuroinflamm. 13, 145 (2016).

16. Huang, W. C. et al. Glycogen synthase kinase-3 negatively regulates antiinflammatory interleukin-10 for lipopolysaccharide-induced iNOS/NO biosynthesis and RANTES production in microglial cells. Immunology $\mathbf{1 2 8}$, e275-e286 (2009) 
17. Eschbach, J. et al. Mutual exacerbation of peroxisome proliferator-activated receptor gamma coactivator 1alpha deregulation and alpha-synuclein oligomerization. Ann. Neurol. 77, 15-32 (2015).

18. Kozikowski, A. P. et al. Highly potent and specific GSK-3beta inhibitors that block tau phosphorylation and decrease alpha-synuclein protein expression in a cellular model of Parkinson's disease. ChemMedChem 1, 256-266 (2006).

19. Liu, L. et al. Adipose-specific knockout of SEIPIN/BSCL2 results in progressive lipodystrophy. Diabetes 63, 2320-2331 (2014).

20. Li, G. et al. Seipin knockout in mice impairs stem cell proliferation and progenitor cell differentiation in the adult hippocampal dentate gyrus via reduced levels of PPARgamma. Dis. Model Mech. 8, 1615-1624 (2015).

21. Assayag, K., Yakunin, E., Loeb, V., Selkoe, D. J. \& Sharon, R. Polyunsaturated fatty acids induce a-synuclein-related pathogenic changes in neuronal cells. Am. J. Pathol. 171, 2000-2011 (2007).

22. Manning-Bog, A. B. et al. The herbicide paraquat causes up-regulation and aggregation of alpha-synuclein in mice: paraquat and alpha-synuclein. J. Biol. Chem. 277, 1641-1644 (2002).

23. Ebihara, C. et al. Seipin is necessary for normal brain development and spermatogenesis in addition to adipogenesis. Hum. Mol. Genet. 24, 4238-4249 (2015).

24. Yakunin, E. et al. Alpha-synuclein neuropathology is controlled by nuclear hormone receptors and enhanced by docosahexaenoic acid in a mouse model for Parkinson's disease. Brain Pathol. 22, 280-294 (2012).

25. Perrin, R. J. Exposure to long chain polyunsaturated fatty acids triggers rapid multimerization of synucleins. J. Biol. Chem. 276, 41958-41962 (2001).

26. Sharon, $\mathrm{R}$. et al. The formation of highly soluble oligomers of alpha-synuclein is regulated by fatty acids and enhanced in Parkinson's disease. Neuron 37, 583-595 (2003).

27. Kragh, C. L. et al. Alpha-synuclein aggregation and Ser-129 phosphorylationdependent cell death in oligodendroglial cells. J. Biol. Chem. 284 10211-10222 (2009)

28. Duka, T., Duka, V., Joyce, J. N. \& Sidhu, A. Alpha-synuclein contributes to GSK3beta-catalyzed Tau phosphorylation in Parkinson's disease models. FASEB J. 23, 2820-2830 (2009).

29. Credle, J. J. et al. GSK-3beta dysregulation contributes to parkinson'slike pathophysiology with associated region-specific phosphorylation and accumulation of tau and alpha-synuclein. Cell Death Differ. 22, 838-851 (2015).
30. Mou, Z. et al. Bibenzyl compound 20c protects against endoplasmic reticulum stress in tunicamycin-treated PC12 cells in vitro. Acta Pharmacol. Sin. 37, 1525-1533 (2016).

31. Sugeno, N. et al. Serine 129 phosphorylation of alpha-synuclein induces unfolded protein response-mediated cell death. J. Biol. Chem. 283 23179-23188 (2008)

32. Chen, M. et al. Tea polyphenols alleviate motor impairments, dopaminergic neuronal injury, and cerebral a-synuclein aggregation in MPTP-intoxicated parkinsonian monkeys. Neuroscience 286, 383-392 (2015).

33. Shoji, H., Takao, K., Hattori, S. \& Miyakawa, T. Age-related changes in behavior in C57BL/6J mice from young adulthood to middle age. Mol. Brain 9, 11 (2016).

34. Hirsch, E. C. \& Hunot, S. Neuroinflammation in Parkinson's disease: a target for neuroprotection? Lancet Neurol. 8, 382-397 (2009).

35. Zhao, $X$. et al. Neuronal PPARgamma deficiency increases susceptibility to brain damage after cerebral ischemia. J. Neurosci. 29, 6186-6195 (2009).

36. Volles, M. J. \& Lansbury, P. T. Jr. Zeroing in on the pathogenic form of alphasynuclein and its mechanism of neurotoxicity in Parkinson's disease. Biochemistry 42, 7871-7878 (2003)

37. Duda, J. E., Lee, V. M. \& Trojanowski, J. Q. Neuropathology of synuclein aggregates. J. Neurosci. Res. 61, 121-127 (2000).

38. Gao, H. M. et al. M. Neuroinflammation and oxidation/nitration of alphasynuclein linked to dopaminergic neurodegeneration. J. Neurosci. 28 7687-7698 (2008)

39. Patki, G. \& Lau, Y.-S. Melatonin protects against neurobehavioral and mitochondrial deficits in a chronic mouse model of Parkinson's disease. Pharmacol. Biochem. Behav. 99, 704-711 (2011).

40. West, M. J. New stereological methods for counting neurons. Neurobiol. Aging 14, 275-285 (1993).

41. Jiang, M. et al. Lack of testicular seipin causes teratozoospermia syndrome in men. Proc. Natl Acad. Sci. USA 111, 7054-7059 (2014).

42. Wittig, I., Braun, H. P. \& Schagger, H. Blue native PAGE. Nat. Protoc. 1, 418-428 (2006).

43. Salehi-Sadaghiani, M. et al. NMDA receptor involvement in antidepressant-like effect of pioglitazone in the forced swimming test in mice. Psychopharmacology 223, 345-355 (2012).

44. Martins, D. F. et al. The antinociceptive effects of AR-A014418, a selective inhibitor of glycogen synthase kinase-3 beta, in mice. J. Pain 12, 315-322 (2011). 